\title{
ADMIT- A Five Dimensional Approach towards Standardization of Network and Computer Attack Taxonomies
}

\author{
Chanchala Joshi \\ Institute of Computer Science \\ Vikram University, Ujjain, M.P. India
}

\author{
Umesh Kumar Singh \\ Institute of Computer Science \\ Vikram University, Ujjain, M.P. India
}

\begin{abstract}
This paper proposed a five dimensional taxonomy ADMIT which captures five major classifiers to characterize the nature of attacks. These are classification by attack vector, classification by defense, classification by method, classification by impact and classification by attack target. The classification structure of proposed taxonomy described the nature of attacks thoroughly. The administrator can use the proposed taxonomy to locate strategies that are appropriate for securing their system against vulnerabilities that can be exploited. Use of ADMIT taxonomy in network defense strategies can improve the overall level of security.
\end{abstract}

\section{Keywords}

Network security; Vulnerability; Attack taxonomy

\section{INTRODUCTION}

Security threats to computers and networks have been a problem since computers and networks were firstly used. With the rapid growth of the Internet, attacks are no longer limited in computer alone. They have created a global threat, causing great damages in individuals, communities and national security. Network attacks are almost a subset of computer attacks but some network attacks are outside the computer attack domain [1]. For security assessment it is necessary to find and classify these attacks. The first step in understanding attacks is to classify them into a taxonomy based on their characteristics. Taxonomy classifies attack into well-defined and easily understood categories. Such classification can be used for performing a systematic security assessment of a system.

Taxonomy provides a way to know about attacks at a level higher than a simple list of vulnerabilities. It provides a classification system that ideally suggests ways to mitigate attacks by prevention, detection and recovery. It can aid risk management by identifying vulnerabilities and making attacker characteristics explicit. Ideally its insights can predict future attacks by exposing unguarded areas. Every attack is performed by someone and every attacker has an identity and the motive of the attack is to do certain thing. An attack targets some service or layer exploiting vulnerability. Each of these attack elements or say dimensions is necessary to understand which includes the whole process of attack. Therefore useful and standard taxonomy should answer the following questions:

i. Who is the attacker?

ii. How to face the attack?

iii. How is it attacked?

iv. What are the results?

v. What is the target?

The proposed taxonomy ADMIT answers the each question in turn. Taken altogether the attack vector, defense, method, impact and the target, describe the nature of attack. ADMIT provides useful information to the network administrator. This paper provides a mean to classify vulnerabilities with their impact and also with defensive strategies.

\section{MOTIVATION}

One of the major problems in computer and network system security assessment is lack of standard vulnerability categorization scheme called taxonomy. A standard vulnerability categorization scheme also aids in finding general trends which are responsible for existence of vulnerabilities. Many of the attempts have been made in this direction in the past but still this issue is unresolved. The overall objective of this research work is to analyze different categories of prominent vulnerability taxonomies to identify the level of abstraction and common factors for standardization of network vulnerability taxonomy.

\section{RELATED WORK}

One of the first taxonomies to be developed was given in RISOS (Research In Secure Operating Systems) project [3]. The RISOS security taxonomy was based on flaws found in three operating systems: IBM's OS/MVT for the IBM 360, UNIVAC's 1100 Series operating system and Bolt Beranek and Newman's TENEX system for the PDP-10. The classification consisted of seven categories. The main contribution of this study was the classification of integrity flaws found in operating systems. It also led to classify the same flaw in multiple categories

Protection Analysis (PA) Taxonomy [4] was one of the earliest to address security concerns. The objective of the PA project was to provide a basis for categorizing protection errors according to their security relevant properties using an automated and patternmatching approach. This taxonomy was based on 100 flaws found in six different operating systems. It had four global categories: improper protection (initialization and enforcement), improper validation, improper synchronization and improper choice of operand or operation. The categories in this taxonomy were broad and the same flaw was classified into multiple categories. The contribution of this study was the introduction of several types of security flaws like allocation or deallocation of residuals and serialization errors that remained relevant.

Aslam defined a classification of security faults $[5,6]$ in the Unix Operating System. He focused on UNIX operating system flaws only and presented three main categories: Operational fault, Environmental fault and Coding fault. Coding faults, comprising faults introduced during software development and Operational faults, resulting from improper installation of software, unexpected integration incompatibilities, or when a programmer fails to completely understand the limitations of the run-time modules.

Krsul [7] extends Aslam's work and developed a detailed taxonomy. Main categories proposed in this taxonomy were: Design, Environmental assumptions, Coding faultsand Configuration errors. In proposed scheme, there is ambiguity in distinguishing between objects and attributes because of 
interpretation scope permitted by taxonomy. It also fails to elaborate on how assumptions lead to vulnerabilities.

Bishop [8] analyzed the RISOS, PA and Aslam's taxonomies and showed that these classes could be mapped onto each other. Bishop presents taxonomy of UNIX vulnerabilities by classifying them with explicit goal of describing a technique to find vulnerabilities. Bishop's work focused on categorizing security vulnerabilities in software to assist security practitioners in maintaining more secure systems through an understanding of these vulnerabilities. John Howard [9] extended this idea in his work in which he analyzed and classified 4299 security related incidents on the internet. Howard's work was notable because he included attackers, results and objectives as classification categories expanding threat taxonomies beyond the technical details of an attack to include more intangible factors such as an attacker's motivation for conducting an attack.

Kjaerland's [10] study categorized cyber intrusions based on four categories; (1) method of operations, (2) impact of the intrusion, (3) source of the intrusion and (4) target. This study examined the likelihood of attacks against different kinds of targets and the likelihood of various kinds of attacks occurring together on a given target.

Lough [11] proposed an attack-centric taxonomy called VERDICT (Validation, Exposure Randomness, Deallocation, Improper Conditions Taxonomy). Lough focuses on four major causes of security errors: Improper Validation, Improper Exposure, Improper Randomness and Improper Deallocation. Validation refers to improperly validating or unconstrained data which also includes physical security. Exposure involves the improper exposure of information that could be used directly or indirectly for the exploitation of vulnerability. Randomness deals with the fundamentals of cryptography and the improper usage of randomness. Deallocation is the improper destruction of information or residuals of data which also includes dumpster diving. He uses one or more of these characteristics to describe vulnerability within a system. Hansman and Hunt [1] describe Lough's taxonomy as lacking pertinent information that would be beneficial for knowledge bodies such as CERT, to classify day-to-day attacks and issuing advisories. Lough's taxonomy lacks the classification to the type of attack, such as worms, Trojans, viruses, etc.

Chris Simmons [2] created a cyber-attack taxonomy called AVOIDIT which described attacks using five, extensible classifications: Attack Vector, Operational Impact, Defense,
Informational Impact and Target. This taxonomy was created as a network taxonomy which unlike previous efforts, allowed the classification of blended attacks. Additionally, it also allowed for the classification of attacks by both operational and informational impacts and was designed to help educate defenders by looking at attacks' various impacts, vectors or target types. While this taxonomy focused exclusively on cyber-attacks, its structure and style were very useful in designing the proposed taxonomy in this paper, especially the ability to view and categorize attacks from Applegate different taxonomic perspectives.

Scott D. [12] proposed cyber conflict taxonomy. Subjects of the taxonomy were entered as either events or entities and then categorized using the categories and subcategories of actions or actors. Each of these categories then further subdivided into increasingly specific subcategories used to describe the defining characteristics of each subject and labeled lateral linkages are used to illustrate the associative relationships between entities and events. The categories were organized in both a hierarchical and associative manner to illustrate the relationships between subjects and categories.

\section{PROPOSED TAXONOMY-ADMIT}

Development of a successful taxonomy is required to meet some standard properties. E. G. Amoroso [13] lists the well accepted principles of a good classification as: Public acceptance, Comprehensibility, Completeness, Determinism, Mutual exclusion, Repeatability, Unambiguous and Useful. A good taxonomy should adhere to these properties. Applying these requirements for a complete taxonomy a standard taxonomy ADMIT is proposed to classify network and computer vulnerability.

The proposed taxonomy ADMIT uses five major classifiers as five dimensions to characterize the nature of an attack, which are classification by attack vector, classification by defense, classification by method, classification by impact and classification by attack target. The first dimension is used to categorize the attack based on attack vector, a path by which an attacker can access to a host. The second dimension covers the defense, the action taken to protect the system from attack. Method of operation is covered in third dimension, which refers to the methods used by perpetrator to carry out an attack. Fourth dimension covers the impact, the effect of the attack; and the attack target is covered in the fifth dimension.

Fig1 provides an overview of proposed taxonomy. 


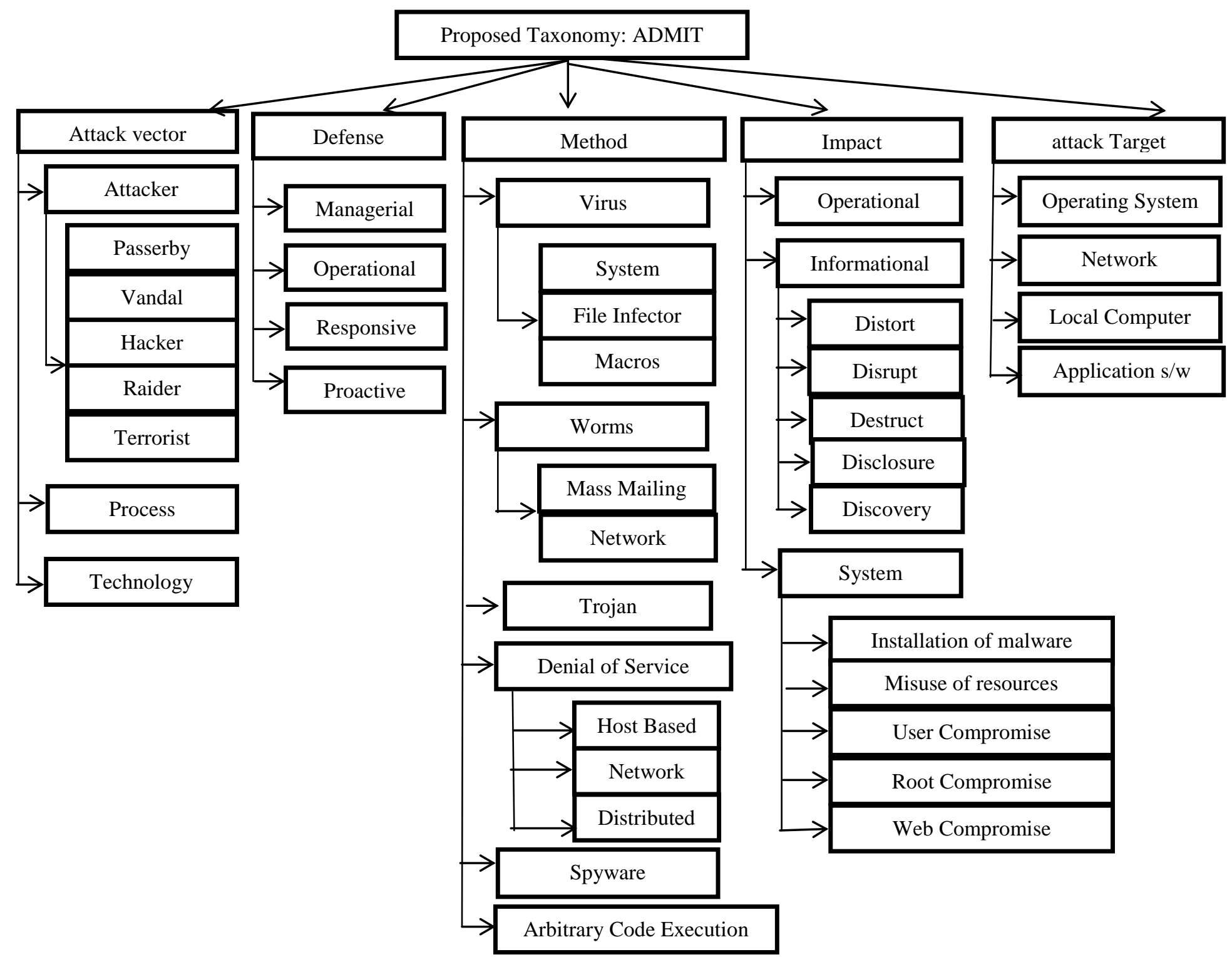

Fig1. ADMIT: An Approach towards Network and Computer attacks

\subsection{Classification by Attack Vector}

An attack vector describes the path or means by which an attacker attempts to gain access to a network or computer system. This category has been further divided into three sub vectors which are attackers, processes and technology. Each of these subdivisions could be further subdivided into increasingly specific and discrete vector.

\subsubsection{Attacker}

It is valuable to know who the attacker is in so far. As it will imply what they are likely to do and how well they would be able to do it. The attackers consist of a range of types of people who may launch an attack. These range from hackers to terrorists. Anthony [14] characterizes an attacker according to four dimensions: motive, determination, knowledge and resources:

i. Passerby: Motivated by spontaneity; not determined; very little knowledge; few resources.

ii. Vandal: Desires to make damages, perhaps visibility; moderately determined; little knowledge; few resources necessary.

iii. Hacker: Desires access, motivated by curiosity and interest; highly determined; highly knowledgeable; moderate resources. iv. Raider: Driven by personal or organizational monetary and/or political gain; highly determined; moderately .highly knowledgeable; moderate resources.

v. Terrorist or Foreign Power: Causes real-world damage by compromise of critical systems, motivated by enmity; very determined; highly knowledgeable; very well resourced with time, money and man-power.

\subsubsection{Process}

This subcategory describes the vector based on the manipulation of flawed organizational processes. For example suppose an organization allows a visitor to carry their security passes rather than mandating that the credentials be verified directly with the issuing source. Then an attacker might exploit this flawed process to illegitimately gain legitimate credentials to a system.

\subsubsection{Technology}

This category describes a vector based on the manipulation of technology and technical processes. An example would be exploiting vulnerability in a software program. 


\subsection{Classification by Defense}

This category describes the action taken to protect the system from attack. National Institute of Standard and Technology divided the defense into two dimensions: managerial and operational. Scott [12] expanded the NIST standard by adding responsive defense. A new dimension proactive defense is added in this category.

\subsubsection{Managerial}

These are defensive techniques and methods adopted by management. It is like an organization's security strategy.

i. Remove from network: Remove infected hosts, preventing further damage.

\subsubsection{Operational}

These are defensive policies and procedure implemented to improve security of system.

i. White listing: This is a list of permissible connections that are known to the defender.

ii. Reference advertisement: These are the notes provided by the defender to mitigate an attack or a vulnerability database reference number that is used to mitigate the vulnerability or attack.

\subsubsection{Responsive}

Under this take the appropriate step to correct the situation during exploitation

i. Patch System: [2] Applying patches the vendor has released due to some vulnerability within software in use. When a vulnerability or attack is present, a defender fails to utilize the patches a vendor provides.

ii. Correct Code: [2] An organization may release a code patch to specific application that will close the potential for an attacker to exploit.

\subsubsection{Proactive tool}

These are defensive techniques or strategies executed by automated system to improve the security of system.

\subsection{Classification by Method}

This classification refers to the way used by an intruder to carry out an attack. It also tells about the tools used and also about the access point.

\subsubsection{Virus}

A virus is a piece of code that while running will attach itself with other programs which will run again when those programs are running.

\subsubsection{Worms}

This is a program that propagates itself by attacking other machines and copying itself with them.

\subsubsection{Trojans}

It is a program that adds subversive functionality to an existing program.

\subsubsection{Denial of Service}

Denial of service (DoS) is an attack to deny a victim access to a particular resource or service. It has become one of the major threats and has been rated among the hardest Internet security issues [15]. This subcategory can be further classified into following: i. Host Based - A Host based DoS aims the attack at specific computer target within the configuration, operating system or software of a host. These types of attacks usually involved resource hogs that aim at consuming all resources on computer crashers which attempts to crash the host system [15].

ii. Network Based - A network based DoS targets the complete network of computers to prevent the network providing normal services. Network based DoS usually occurs in the form of flooding with packets where the network's connectivity and bandwidth are the targets [15].

iii. Distributed - A Distributed Denial of Service (DDoS) is becoming more popular as an attacker's choice of DoS. Distributed denial of service uses multiple attack vectors to obtain its goal [15].

\subsubsection{Spyware}

It is a type of malware program that is covertly installed and infects its target by collecting information from a computing system without owner's consent.

\subsubsection{Arbitrary Code Execution}

It involves a malicious entity that gains control through some vulnerability injecting its own code to perform any operation the overall application has permission [15].

\subsection{Classification by Impact}

\subsubsection{Operational Impact}

This category describes the impact of an intrusion on victim's operations.

i. Organizational disruption: Impact of an intrusion which causes the disruption of operations within an organization. An example is altering information in a supplier database system to reroute critical supplies to the wrong destinations.

ii. Loss of competitive advantage: It is due to disclosure of plans or confidential data.

\subsubsection{Informational Impact}

[2] This category describes the impact an intrusion has directly on victim's information.

i. Distort: It's a distortion of information. It usually happens when an attack has caused a modification of life.

ii. Disrupt: It is usually from Denial of Service. When an attack involves disruption, it is an access change or removal of access to victim or information.

iii. Destruct: Destruction is called when an attack has caused a deletion of life or removal of access.

iv. Disclosure [12]: A disclosure of information provides a view of information to the attacker of which they would not have access to.

v. Discovery: It is related to discover information that is not previously known.

\subsubsection{Organization's System Impact:}

This category describes the impact of an intrusion on the actual system of victim's organization.

i. Installation of malware: The installation of malicious software onto the target host or system.

ii. Misuse of resources: An unauthorized use of system resources.

iii. User Compromise: A perpetrator gaining unauthorized use of user privileges on a host, as a user compromise [10].

iv. Root Compromise: Gaining unauthorized privileges of an administrator on a particular host [10]. 
v. Web Compromise: A website or web application using vulnerabilities to further an attack [10]. An attack can occur through a web compromise, usually via cross site scripting or sql injection

\subsection{Classification by Attack Target}

Fifth dimension covers the attack target. Various attacks target a variety of hosts.

\subsubsection{Operating System}

An attack can be formulated to target vulnerabilities within a particular operating system

\subsubsection{Network}

Target a particular network or gain access through a vulnerability within a network or one of the network protocols.

\subsubsection{Local Computer}

It includes an attack targeting user's local computer.

\subsubsection{Application Software}

It includes an attack towards specific software. An application can be either client or server. A client application is software that is available to aid a user performing common tasks. A server application is software designed to serve as a host to multiple concurrent users [2].

\section{CLASSIFICATION STRUCTURE OF ADMIT}

Classification structure describes how the proposed taxonomy can be practically applied. Also the classification structure evaluates and analyzes the proposed taxonomy.

Table 1 shows the classification of ADMIT taxonomy.

Table 1: classification by ADMIT taxonomy

\begin{tabular}{|c|c|c|c|c|c|}
\hline Attack Name & Attack Vector & Defense & Method & Impact & Target \\
\hline Blaster & Buffer Overflow & $\begin{array}{l}\text { White listing } \\
\text { Patch System }\end{array}$ & $\begin{array}{c}\text { Network Aware } \\
\text { Worm }\end{array}$ & Distort & $\begin{array}{l}\text { Windows NT } \\
4.0, \mathrm{XP}, 2000\end{array}$ \\
\hline Chernobyl & Misconfiguration & $\begin{array}{c}\text { Reference } \\
\text { Advertisement }\end{array}$ & $\begin{array}{l}\text { Virus: File } \\
\text { infector }\end{array}$ & Disrupt & $\begin{array}{c}\text { Operating } \\
\text { System }\end{array}$ \\
\hline Code Red & Buffer Overflow & Patch System & $\begin{array}{c}\text { Worm: Network } \\
\text { aware }\end{array}$ & Discovery & Network \\
\hline $\begin{array}{l}\text { Debian } \\
\text { Admin }\end{array}$ & Kernel flaw & Patch System & $\begin{array}{l}\text { Virus: System } \\
\text { Infector }\end{array}$ & $\begin{array}{c}\text { Root compromise } \\
\text { Disrupt }\end{array}$ & $\begin{array}{c}\text { Operating } \\
\text { System }\end{array}$ \\
\hline Infector & Design flaw & $\begin{array}{c}\text { Reference } \\
\text { Advertisement }\end{array}$ & $\begin{array}{l}\text { Worm: File } \\
\text { infector }\end{array}$ & Distort & $\begin{array}{c}\text { Operating } \\
\text { System: DOS }\end{array}$ \\
\hline Melissa & $\begin{array}{c}\text { Technology: } \\
\text { Misconfiguration }\end{array}$ & Patch System & $\begin{array}{l}\text { Worm: Mass } \\
\text { mailing }\end{array}$ & Disrupt & $\begin{array}{c}\text { Application: } \\
\text { MS Word } \\
97,2000\end{array}$ \\
\hline $\begin{array}{c}\text { MS RPC } \\
\text { Stack } \\
\text { Overflow }\end{array}$ & $\begin{array}{l}\text { Buffer } \\
\text { Overflow: } \\
\text { Stack }\end{array}$ & $\begin{array}{l}\text { Installed Malware: } \\
\text { ACE }\end{array}$ & $\begin{array}{c}\text { Reference } \\
\text { Advisement } \\
\text { Patch System }\end{array}$ & Distort & $\begin{array}{c}\text { OS: } \\
\text { Windows } \\
\text { Server }\end{array}$ \\
\hline Nimda & Misconfiguration & $\begin{array}{l}\text { White listing } \\
\text { Reference } \\
\text { Advertisement }\end{array}$ & $\begin{array}{l}\text { File Infector, } \\
\text { Trojan \& DoS }\end{array}$ & Disrupt & $\begin{array}{l}\text { Application: } \\
\text { IE }\end{array}$ \\
\hline Slammer & $\begin{array}{c}\text { Technology: } \\
\text { Misconfiguration }\end{array}$ & Patch system & $\begin{array}{l}\text { Worm: Installed } \\
\text { malware }\end{array}$ & Discovery & Network \\
\hline Yamanner & $\begin{array}{l}\text { Technology: Design } \\
\text { flaw }\end{array}$ & $\begin{array}{c}\text { Reference } \\
\text { Advertisement }\end{array}$ & $\begin{array}{l}\text { Worm: Installed } \\
\text { malware }\end{array}$ & Disrupt & $\begin{array}{l}\text { Local } \\
\text { computer }\end{array}$ \\
\hline
\end{tabular}

\section{EVALUEATION OF ADMIT}

In this section the proposed taxonomy ADMIT is compared with past taxonomies described in section 1.2. The comparison result represents that how successfully it captures vulnerability attack information and provides a defender countermeasures that can be efficient in preventing attacks.

\section{$6.1 \quad$ Blaster}

The Blaster Worm was a computer worm that spread on computers running the Microsoft operating systems Windows XP and Windows 2000, during August 2003 [18].

In Table 2 comparison of Blaster attack with other prominent taxonomies is shown. 
Table 2: Blaster attack classification

\begin{tabular}{|c|c|c|c|c|c|c|c|c|}
\hline \multicolumn{9}{|c|}{ Lough's taxonomy: VERDICT } \\
\hline Attack Name & \multicolumn{2}{|c|}{ Improper Validation } & \multicolumn{3}{|c|}{ Improper Exposure } & \multicolumn{2}{|c|}{ Improper Randomness } & Improper Deallocation \\
\hline Blaster & \multicolumn{2}{|c|}{$\mathrm{X}$} & \multicolumn{3}{|c|}{$\mathrm{X}$} & & & \\
\hline \multicolumn{9}{|c|}{ Howard's taxonomy } \\
\hline Attack Name & Tools & \multicolumn{2}{|c|}{ Vulnerability } & \multicolumn{2}{|c|}{ Action } & \multicolumn{2}{|c|}{ Target } & Unauthorized Result \\
\hline Blaster & Program & \multicolumn{2}{|c|}{ Buffer Overflow } & \multicolumn{2}{|c|}{ Modify } & \multicolumn{2}{|c|}{ Network } & Corruption of Information \\
\hline \multicolumn{9}{|c|}{ Hansman and Hunt's taxonomy } \\
\hline Attack Name & \multicolumn{2}{|c|}{$1^{\text {st }}$ Dimension } & \multicolumn{2}{|c|}{ 2nd Dimension } & \multicolumn{2}{|c|}{ 3rd Dimension } & \multicolumn{2}{|r|}{ 4th Dimension } \\
\hline Blaster & \multicolumn{2}{|c|}{ Network - Aware Worm } & \multicolumn{2}{|c|}{ Network } & \multicolumn{2}{|c|}{ CAN-2003-0352 } & \multicolumn{2}{|c|}{ TCP packet flooding DoS } \\
\hline \multicolumn{9}{|c|}{ Proposed taxonomy : ADMIT } \\
\hline Attack Name & \multicolumn{2}{|c|}{ Attack vector } & ense & \multicolumn{3}{|c|}{ Method } & Impact & Target \\
\hline Blaster & \multicolumn{2}{|c|}{ Buffer Overflow } & $\begin{array}{l}\text { White listing } \\
\text { Patch System }\end{array}$ & \multicolumn{3}{|c|}{ Computer Worm } & Distort & Windows XP, 2000 \\
\hline
\end{tabular}

\subsection{Melissa}

First found on March 26, 1999, Melissa shut down Internet Email systems that got clogged with infected e-mails propagating from the virus. Melissa was not originally designed for harm, but it overloaded servers and caused problems [18].

Table 3: Melissa attack classification
In Table 3, comparison of Melissa attack with other prominent taxonomies is shown.

Lough's taxonomy: VERDICT

\begin{tabular}{|c|l|c|c|c|c|}
\hline Attack Name & \multicolumn{1}{|c|}{ Improper Validation } & Improper Exposure & Improper Randomness & Improper Deallocation \\
\hline Melissa & & \multicolumn{2}{|c|}{ X } & X \\
\hline Howard's taxonomy & Tools & Vulnerability & Action & Target & Unauthorized Result \\
\hline Attack Name & Script & Configuration & Authenticate & Data & Corruption of Information \\
\hline Melissa &
\end{tabular}

Hansman and Hunt's taxonomy

\begin{tabular}{|c|c|c|c|c|c|}
\hline Attack Name & \multicolumn{1}{|c|}{$\mathbf{1}^{\text {st }}$ Dimension } & \multicolumn{1}{|c|}{ 2nd Dimension } & 3rd Dimension & 4th Dimension \\
\hline Melissa & Mass- Mailing Worm & Microsoft Word 97,2000 & Configuration & $\begin{array}{c}\text { Macro Virus \&TCP packet flooding } \\
\text { DoS }\end{array}$ \\
\hline Proposed taxonomy : ADMIT & \multicolumn{1}{|c|}{ Method } & \multicolumn{1}{|l|}{ Impact } & Target \\
\hline Attack Name & Attack vector & Defense & Disrupt & $\begin{array}{l}\text { Application: } \\
\text { Word 97,2000 }\end{array}$ \\
\hline Melissa & Misconfiguration & Patch System & Worm: Mass mailing & MS
\end{tabular}

In Table2, Table 3 evaluation of ADMIT taxonomy by comparing it with other prominent taxonomies is shown. These comparisons conclude that Lough's does not provide useful information in describing the attacks. Howard's taxonomy provides general information. Hansman and Hunt's taxonomy provides more useful information about method of operation, target, vulnerability and payload. The proposed ADMIT taxonomy provides information to network administrator about the cause of attack, possible defense mechanism, method of attack, attack result and target to reduce attack's impact.

\section{LIMITATIONS OF ADMIT}

Attacks have become increasingly present in computer and network system. Provide the ability to prevent all attacks is extremely difficult [17]. Risk factor is an important aspect in achieving security. Although the proposed taxonomy is able to classify the vulnerability thoroughly but there is no such dimension or attack attribute in classification structure associated with attack that directly implies the severity of vulnerability. Further research can be done to include remediation plans to reduce the risks to acceptable level and to address exposures. 


\section{CONCLUSION}

This paper provides a five dimensional taxonomy which captures five major classifiers to characterize the nature of attacks. These are attack vector, defense, method, impact and attack target. The classification structure is able to thoroughly classify vulnerabilities and provides a more apparent approach to educate the defender on possible attacks using vulnerability details. The classification structure of proposed taxonomy provides information to network administrator about the cause of attack, possible defense mechanism, attack method, attack result and target to reduce attack's impact. The administrator can also use the proposed taxonomy to locate strategies that are appropriate for securing their system against vulnerabilities that can be exploited and used for unauthorized access. Use of ADMIT in a network defense strategies can improve the overall level of security. In future research remediation plans will be included to reduce the risks to acceptable level and to address exposures.

\section{REFERENCES}

[1] Hansman, S., Hunt R., "A taxonomy of network and computer attacks". Computer and Security, vol. 24, issue 1, Feb 2005, PP. 31-43.

[2] Simmons, C., Ellis, C., Shiva, S., Dasgupta, D., \& Wu, Q. "AVOIDIT: A Cyber Attack Taxonomy", University of Memphis, Technical Report CS-09-003, 2009. [Online]. Available:

http://issrl.cs.memphis.edu/files/papers/CyberAttackTaxono my IEEE Mag.pdf

[3] R. P. Abbott et al, "Security Analysis and Enhancements of Computer Operating Systems," Report NBSIR 76-1041, Institute for Computer Science and Technology, Natl. Bur. of Stnds, Apr. 1976.

[4] Bisbey, R. and D. Hollingsworth, "Protection Analysis Project Final Report, "Information Sciences Institute, University of Southern California, Marina Del Rey, CA, 1978.

[5] T. Aslam, "A taxonomy of Security Faults in the Unix Operating System," M.S. Thesis, Purdue University, 1995.

[6] T. Aslam, "Use of a taxonomy of Security Faults," Technical Report 96-05, COAST Laboratory, Department of Computer Science, Purdue University, March 1996.
[7] I. Krsul, "Software Vulnerability Analysis," Ph.D. dissertation, Purdue Univ., 1998.

[8] M. Bishop, "A Taxonomy of UNIX System and Network Vulnerabilities," Technical Report CSE-95-10, Purdue University, May 1995.

[9] Howard, John D. and Longstaff, Thomas A. "A Common Language for Computer Security Incidents," Technical report, Sandia National Laboratories, Oct. 1998.

[10] Kjaerland, M., "A taxonomy and comparison of computer security incidents from the commercial and government sectors". Computers and Security, Volume 25, Issue 7, October 2006, PP 522-538.

[11] Lough, Daniel. "A Taxonomy of Computer Attacks with Applications to Wireless Networks," PhD thesis, Virginia Polytechnic Institute and State University, 2001.

[12] Scott D., Angelos S," Towards a Cyber Conflict Taxonomy", 5th International Conference on Cyber Conflict K. Podins, J. Stinissen, M. Maybaum (Eds.), 2013.

[13] E. G. Amoroso, "Fundamentals of Computer Security Technology", Upper Saddle River, NJ: Prentice-HallPTR, 1994.

[14] Anthony D. Wood, John A. Stankovic, "A Taxonomy for Denial-of-Service Attacks in Wireless Sensor Networks," Handbook of Sensor Networks: Compact Wireless and Wired Sensing Systems, CRC Press, 2004.

[15] C. Douligeris and A. Mitrokotsa, "DDoS Attacks and Defense Mechanisms: Classification and State-of-theart," Comp. Networks, Volume 44, Issue 5, April 2004, PP 643666

[16] David Moore, Vern Paxson, Stefan Savage, Colleen Shannon, Stuart Staniford and Nicholas Weaver. "Inside the slammer worm", IEEE Security and Privacy, volume 1, 2003, PP 33-39.

[17] William A. Arbaugh, William L. Fithen, and John McHugh, "Windows of Vulnerability: A Case Study Analysis", IEEE Computer, 33, issue 12, Dec. 2000, PP 52-59.

[18] CERT Coordination Center, "CERT Advisory CA-2003-20 W32/Blaster worm," Aug. 2003; www.cert. org/advisories/CA-2003-20.html. 